\title{
Interoceptive perception of optogenetic brain stimulation
}

\author{
Authors: Jorge Luis-Islas ${ }^{1,2}$, Monica Luna ${ }^{1}$, Benjamin Floran ${ }^{2}$, and Ranier Gutierrez ${ }^{1 *}$
}

\section{Affiliations:}

${ }^{1}$ Laboratory of Neurobiology of Appetite. Department of Pharmacology, Center for Research and Advanced Studies of the National Polytechnic Institute, CINVESTAV, Mexico City, Mexico

${ }^{2}$ Department of Physiology, Biophysic, and Neuroscience, Center for Research and Advanced Studies of the National Polytechnic Institute, CINVESTAV, Mexico City, Mexico

*Correspondence to: ranier@cinvestav.mx.

Abstract: How do animals experience brain manipulations? Optogenetics has allowed us to manipulate selectively and interrogate neural circuits underlying brain function in health and disease. However, in addition to their evoked physiological functions, it is currently unknown whether mice could perceive arbitrary optogenetic stimulations. To address this issue, mice were trained to report optogenetic stimulations to obtain rewards and avoid punishments. It was found that mice could perceive optogenetic manipulations regardless of the brain area modulated, their rewarding effects, or the stimulation of glutamatergic, GABAergic, and dopaminergic cell types. We named this phenomenon optoception. Our findings reveal that mice's brains are capable of "monitoring" their self-activity via interoception, opening a new way to introduce information to the brain and control brain-computer interfaces.

One Sentence Summary: Brain manipulations are perceived

\section{Main Text}

The brain can integrate and sense signals arising from inside the body monitoring the status of the internal milieu in a process named interoception $(1,2)$. This process which is not restricted to visceral stimuli occurs both consciously and non-consciously and comprises all body organs, including the brain $(1,3)$. It is well established that electrical stimulation of various areas can produce a cornucopia of behavioral changes (4-9). However, all these effects had been attributed to their evoked sensory, motor, or rewarding physiological functions. Thus, it is currently unknown whether mice could learn solely from interoceptive signals arising from stimulating the brain itself. To this aim, several behavioral protocols were designed to test whether mice could use optogenetic brain stimulations as a cue to obtain a reward and avoid punishment.

\section{Mice could perceive optogenetic perturbations}

We hypothesized that irrespective of cell type or brain region perturbed optogenetic stimulations would induce a stimulus that animals would perceive and learn to use as a cue to obtain rewards and avoid punishment. To test this hypothesis, we stimulated glutamatergic and GABAergic neurons of the prefrontal cortex (PFC), the afferent fibers of the Nucleus Accumbens (NAc), the GABAergic neurons in the Thalamic Reticular Nucleus (TRN), and the dopaminergic (DA) 
neurons in the Ventral Tegmental Area (VTA). Initially, optical fibers were implanted in the PFC of Thy1-ChR2 mice (PFC ${ }^{\text {Thy } 1}$ ), VGAT-ChR2 mice (PFC $\left.{ }^{\mathrm{VGAT}}\right)$, or wild-type (WT) mice (PFC ${ }^{\mathrm{WT}}$ ). Thy1-ChR2 mice express the channelrhodopsin 2 (ChR2) mainly in pyramidal layer $\mathrm{V}$ excitatory glutamatergic neurons $(10,11)$, whereas VGAT-ChR2 mice express ChR2 in GABAergic ${ }^{\text {VGAT+ }}$ neurons that produce an indirect and extensive inhibition (12). WT mice served as a control (Fig. 1A). We chose the PFC because its glutamatergic and GABAergic neurons play an opposing role in learning and working memory $(13,14)$. Also, because we found that the optogenetic stimulation of these cells produced an opposite modulatory pattern as the laser frequency increased (Figs. S1A$\mathrm{D},(15,16))$. In the Thy1-ChR2 mice, $67 \%$ of PFC's neurons were excited, and $20 \%$ inhibited after laser stimulation at $20 \mathrm{~Hz}$. In contrast, stimulation of GABAergic neurons in the VGAT-ChR2 mice resulted in 3\% of neurons excited, and 50\% inhibited (Fig. S1E). We also stimulated subcortical brain regions, i.e., the NAc of Thy1-ChR2 mice (NAc ${ }^{\text {Thy } 1}$ ) or the TRN in the VGATChR2 mice (TRN ${ }^{\mathrm{VGAT}}$ ). In the NAc ${ }^{\text {Thy } 1}$, we activated the glutamatergic afferents innervating the NAc (16). In contrast, GABAergic neurons in TRN ${ }^{\mathrm{VGAT}}$ mice were stimulated (17). Finally, we also stimulated DA neurons in the VTA by driving the expression of ChR2 in the TH-Cre mice $\left(\mathrm{VTA}^{\mathrm{TH}}, 20\right)$.

Water-deprived mice were initially habituated to alternate licking between two sippers to obtain sucrose (not shown) whereupon they were trained in an optogenetic-cue alternation task, in which $50 \%$ of trials (no-cue) continued alternating between sippers to receive sucrose (Fig. 1B). In contrast, in the other $50 \%$ of trials (cue-trials), mice received halfway between sippers a composed cue (tone $2 \mathrm{kHz}$ + laser $20 \mathrm{~Hz}, 1 \mathrm{~s}$ ), instructing them to stop alternation and return to the previously rewarded sipper to receive sucrose (Correct cue-trial). If mice ignored the cue, i.e., did not change direction and lick the opposite sipper two times, then two airpuffs were delivered as a punishment (Fig. 1B, Error trial; Movie 1). We judged that the mice learned the task if, in 5 consecutive sessions, more than $50 \%$ of cue-trials were correct (Fig. 1C and Fig. S2). We choose a $2 \mathrm{kHz}$ auditory tone because it is barely perceptible to mice (19), resulting in a greater saliency toward the optogenetic stimulation in our task. Of the $10 \mathrm{PFC}^{\mathrm{WT}-2 \mathrm{kHz}}$ mice tested, only 2 solved the task, although they took significantly more sessions to learn than transgenic mice (Fig. 1D). The other $8 \mathrm{WT}$ mice, in some cases even after 130 sessions, never reached the learning criteria (PFC ${ }^{\mathrm{WT}}$ non-

$30 \mathrm{~L}$ trained with a tone $2 \mathrm{kHz}+$ laser; Fig. S3A). We tested 3 of the non-learning mice by increasing the tone from 2 to $10 \mathrm{kHz}$ (which they readily perceive), and all three rapidly reached the learning criterion (Fig. S3B, and Figs. 1C-D, PFC ${ }^{\mathrm{WT}-10 \mathrm{kHz}}$ ). Importantly, all transgenic mice assayed (with tone $2 \mathrm{kHz}+$ laser) learned the task (Fig. S3C), demonstrating that optogenetic brain perturbations can be perceived. To further corroborate that transgenic mice used optogenetic manipulations as a cue, we then removed the $2 \mathrm{kHz}$ tone from the combined cue, and only the laser was delivered. As expected, transgenic mice maintained their performance above learning criteria, even when the laser alone served as a feedback cue (Figs. 1E-F; see block 2 and Movie 2). In contrast, PFC ${ }^{\text {WT }}$ control mice trained with either 2 or $10 \mathrm{kHz}$ tone dropped their performance below chance level after the tone was eliminated, demonstrating that they guided their behavior using the tone, whereas transgenic mice used the optogenetic stimulation as a discriminative stimulus.

We also found that the transgenic mice did not use the blue light as a cue (20) since using a "fake laser" (i.e., they could see the blue laser light outside the skull without receiving optogenetic stimulation, see Movie 3), their task performance significantly decreased (Figs. 1E-F; block 3). 45 We then inquired whether the tone was equally relevant as the laser to solve the task. After 
reacquisition sessions (only laser; second block 2), the laser was replaced for the tone as a cue (Figs. 1E-F; block 4). Unlike the WT mice that increased performance with the tone alone, the transgenic mice exhibited a drastic drop in performance (Movie 4), like that observed in the fake laser condition. Finally, given that in block 4 and last block 1 (Figs. 1E-F), the WT mice exhibited a lower overall performance than in the initial block 1, suggest either that WT mice used some information from the combined cue (tone+light) to guide behavior or more likely it indicates an impairment induced by the extensive testing with the laser alone (i.e., two consecutive blocks 2). In contrast, our results demonstrate that transgenic mice used optogenetic stimulation to guide behavior and completely neglected the tone.

Importantly and to further demonstrate that transgenic mice do not need the tone to learn, we trained a new group of naïve mice but this time only using the laser as a cue. Transgenic mice learned the task equally well, even when only the optogenetic stimulation served as a cue (Fig. S4). These data demonstrate that mice perceived and learn to use solely optogenetic brain stimulations as a cue to solve the task.

\section{Mice could even perceive one single laser pulse}

Having demonstrated that mice could use optogenetic stimulation as a cue, we then tested the importance of the laser stimulation parameters, namely frequency and number of pulses, since the optrode recordings showed that even a single pulse and lower laser frequencies $(\geq 4 \mathrm{~Hz})$ could induce neuronal modulations in both $\mathrm{PFC}^{\text {Thy1 }}$ and $\mathrm{PFC}^{\mathrm{VGAT}}$ mice (Fig. S1). Thus, we trained our mice in two variants of the optogenetic-cue alternation task. Consequently, we randomly varied the laser frequency on a trial-by-trial basis (from 4, 7, 10, 14, and $20 \mathrm{~Hz}$ ). We found that the percent correct responses gradually increased as the frequency approached $20 \mathrm{~Hz}$ (Fig. 2A). In the second task variant, we changed the number of pulses (at $20 \mathrm{~Hz}$ ) and found a gradual performance improvement when the pulses increased (from 1 to 20). What was somewhat unexpected was that mice could even detect a single pulse (Fig. 2B). All regions stimulated showed a similar detection profile with a notable exception of TRN ${ }^{\mathrm{VGAT}}$ mice, which was more sensitive and outperformed in both task variants the other groups. We posit that this arises from its involvement in arousal and attention (21). We conclude that mice could also discriminate between different interoceptive stimuli elicited by distinct optogenetic parameters.

\section{Mice learned two sets of instructions from two different laser frequencies}

We then explored whether mice could learn two different sets of instructions based on the laser frequency delivered to the same brain area. In terms of classical perceptual studies, these instruction sets would correspond to two different task paradigms. First, mice were trained in a laser frequency discrimination task (Fig. 3A), where after visiting the central port, they received either a 10 or $20 \mathrm{~Hz}$ stimulation after which they had to lick in one of the two lateral ports; one frequency signaled the delivery of sucrose in left port and the other that sucrose is in the right port. If they chose the opposite port, they were punished with two airpuffs (Movie 5). Mice learned this task irrespective of the cell type (glutamatergic and GABAergic) and brain region stimulated, the PFC, NAc, and TRN(Fig. 3B). All groups learned in a similar number of sessions (one-way ANOVA; $F(3,17)=2.76, p=0.074)$. However, the $\mathrm{PFC}^{\mathrm{VGAT}}$ group required additional sessions to reach learning criteria $\left(\mathrm{PFC}^{\mathrm{VGAT}}\right.$ vs. both $\mathrm{PFC}^{\text {Thy1 }}$ and $\mathrm{NAc}^{\text {Thy1 }}$, Fisher post hoc test all $p s<0.05$ 
Fig. 3C), which could arise from the detrimental role of prefrontal GABAergic neurons have in working memory (22). Nevertheless, these experiments show that transgenic mice can use optogenetic stimulation as a cue since, in the fake laser session, their task performance was at chance level (Fig. 3D). In another behavioral task, we tested whether mice could categorize laser frequencies. That is, the lower frequencies $(10,12$, and $14 \mathrm{~Hz})$ were rewarded if mice went to the left port, and higher frequencies $(16,18$, and $20 \mathrm{~Hz})$ were rewarded if they went to the right port (Fig. 3E; ports were counterbalanced). We found that transgenic mice choose more the "high" port as the laser frequency increased (Fig. 3F). Thus, mice could also categorize distinct optogenetic laser frequencies.

\section{Optoception does not require that the optogenetic stimulation be rewarding}

It is well-known that rats could guide behavior by using the rewarding effects evoked by electrically stimulating the medial forebrain bundle (8). For this reason, mice were trained in an operant self-stimulation task to demonstrate that the rewarding effects are important but not essential for optoception. In this task, mice had to press an active lever to trigger 1s laser stimulation (Fig. 4A). Only PFC ${ }^{\text {Thy } 1}$ and $\mathrm{NAc}^{\text {Thy } 1}$ mice self-stimulated by pressing the active lever, thereby indicating the stimulation was rewarding (16). In contrast, optogenetic stimulation was not rewarding for control $\mathrm{PFC}^{\mathrm{WT}}$, GABAergic PFC ${ }^{\mathrm{VGAT}}$, and TRN ${ }^{\mathrm{VGAT}}$ mice (Fig. 4B) even though all groups performed equally well in the optogenetic-cue alternation task (Fig. 4C, red dots). These findings were further confirmed in a closed-loop open field task where mice received an optogenetic stimulation every time they crossed the center of an open field (Fig. 4D). As expected, WT mice rarely visited the center of the open field (24). However, PFC ${ }^{\text {Thy }}{ }^{\text {somas stimulation and }}$ activation of its glutamatergic afferent inputs into the NAc ${ }^{\text {Thyl }}(17)$ increased the time spent crossing the center when this zone triggered optogenetic self-stimulation but not during extinction sessions (Figs. 4E-F). In contrast, stimulation of GABAergic somas in both PFC $\mathrm{VGAT}^{\mathrm{V}}$ and $\mathrm{TRN}^{\mathrm{VGAT}}$ mice was neutral; i.e., rewarding or aversive effects were not observed (Fig. S5)(25). Thus, despite that not all optogenetic stimulations were rewarding, they all equally served as a cue to guide behavior (Fig. 4C, red dots).

\section{Activating or silencing a single cell type both serve as optoceptive cue}

35 In another test to determine if mice could perceive optogenetic stimuli, we explored if they could learn optoception from activating or silencing the same cell type. This hypothesis was tested using the Vgat-ires-cre mice to drive the selective expression of ChR2 or Archaerhodopsin (ArchT) in GABAergic neurons in the Lateral Hypothalamus (LH; Fig. 5A; $\mathrm{LH}^{\mathrm{ChR} 2}$ and $\mathrm{LH}^{\mathrm{ArchT}}$, respectively). Thus, we could activate LH GABAergic neurons with ChR2 or silence them with the outward proton pump, ArchT (26). We found that these mice types could learn to use both the optogenetic activation and silencing as a cue to solve the optogenetic-cue alternation task (Fig. 5B). However, stimulating LH GABAergic neurons induced a faster (Fig. 5B; unpaired $\mathrm{t}$-test, $\mathrm{t}_{(11)}=$ 3.774, $p<0.01$ ) and better performance than silencing them (Fig. 5C; two-way ANOVA, interaction mice $\mathrm{x}$ blocks, $\mathrm{F}(6,305)=35.4, p<0.0001)$. Nevertheless, these mice also maintained their 45 performance above the chance level once the tone $2 \mathrm{kHz}$ was removed from the combined cue (i.e., they received the laser alone; Fig. 5C; block 2). In contrast, their performance dropped to chance 
level when they were tested with a "fake laser" (Fig. 5C; block 3 ) or when only the tone was delivered as a cue (Fig. 5C; block 4). Taken together, these results demonstrate that these mice neglected the $2 \mathrm{kHz}$ tone as a cue and used the interoceptive stimuli induced by optogenetic manipulations to guide behavior. We choose LH GABAergic neurons because it is established that they could induce opposing behavioral effects $(27,28)$. For example, in a real-time place preference task, we corroborated that soma stimulation of GABAergic neurons $\left(\mathrm{LH}^{\mathrm{ChR} 2}\right)$ is rewarding (Figs. 5D and E) in the sense that they preferred the side paired with the laser stimulation, whereas silencing GABAergic neurons $\left(\mathrm{LH}^{\mathrm{ArchT}}\right)$ was aversive in that mice avoid the side paired with the laser (Figs. 5D and E). Opposing effects on feeding behavior were also observed in LH neurons (Figs. 5F and G). That is, in sated mice, stimulation of LH GABAergic neurons promoted consumption (Fig. 5F; $\mathrm{LH}^{\mathrm{ChR} 2}$ ), whereas in water-deprived mice, silencing them reduced sucrose intake (Fig. 5G; LH $^{\text {ArchT }}$, and Fig. S6). In sum, although activating (or silencing) LH GABAergic neurons had opposing effects on reward and feeding, both manipulations were perceived and used as feedback cues to guide behavior.

\section{Discussion}

The ability to manipulate the activity of genetically defined cell types via optogenetics has been a game-changing technology in neuroscience. However, very little is known about their sensoryevoked effects. Our findings collectively reveal that mice were capable of perceiving arbitrary optogenetic stimulations. We found that both activation and silencing of various cell types and brain regions were detected and actively reported by mice. Mice could even sense a single laser pulse, discriminate, and categorize between distinct laser frequencies. Moreover, optoception occurred even when optogenetic activation or silencing of the same cell-type elicited rewarding or aversive effects or whether it promoted feeding or stopped it, respectively. We proposed that mice perceive the particular interoceptive state evoked by the brain circuit recruited by the optogenetic manipulation and then learn to use it as a sensory feedback cue to guide behavior. This aligns well with the findings of Doty 1965, who trained monkeys to report electrical stimulation by pressing a lever to obtain a reward and avoid an electric shock on the leg or tail (29), as well as those of Mazurek \& Shieber 2017 who asked them to discriminate the location site of intracranial stimulation delivered in premotor cortex (6). In both cases, monkeys could detect when and whereinto the premotor cortex the stimulation was delivered. Our results further extend these observations to optogenetic manipulations most often employed. In sum, optogenetic manipulations induced interoceptive signals that lead mice to be aware of their brain stimulation. Given that the primary goal of optogenetics is to determine the physiological function (necessity and sufficiency) that a given cell type supports, our results reveal an unexpected but important side effect evoked by most if not all optogenetic perturbations. Thus, more attention should be paid to the sensory-evoked effects induced by optogenetics.

How do humans and mice experience brain manipulations -electrical or optogenetic- is an intriguing question. Are they experienced as a natural or artificial stimulus? One can argue that co-activation of an arbitrarily large number of neurons rarely occurs under physiological conditions (with optogenetics, this effect is perhaps exacerbated since only one specific cell type 45 and associated brain circuits are co-activated). Thus, it would be most likely experienced as artificial (30). However, more focalize intracranial microstimulation of the somatosensory cortex 
(S1) seems to be experienced more naturally since it could even be substituted for a natural sensory stimulus $(5,31)$. Of course, those studies could neither rule out some degree of embodiment (30). The seminal work of Wilder Penfield revealed that electrical stimulation could induce a "brainbow" of effects comprising noticeable movements, urge to move, somatosensory, visual, or auditory percepts, skin tingling or numbness, as well as rewarding or aversive effects, and even complex emotions (32). However, in other instances produced no identifiable effect (32). Furthermore, various optogenetic cell type stimulations of the S1 cortex and its projections bias (i.e., potentiate or suppress) somatosensory perception, whereas others did not affect perception at all (33). Based on this in our experiments, it would be unlikely that mice felt precisely the same interoceptive sensation in each optogenetic manipulation tested. Thus, optogenetic manipulations may also be useful to investigate specific interoceptive states evoked by various cell types, akin to the interoceptive conditioning phenomenon (34) elicited by drug-induced body states $(35,36)$. Nevertheless, our results suggest that the rewarding, neutral, or aversive effects induced by optogenetic manipulations are not necessary to experience optoception since mice equally learn to used all three interoceptive states as a conditioned stimulus. We posit that mice would be aware of most, if not all, optogenetic brain stimulations, probably using interoception.

Thus, our results demonstrate that the brain is capable of "monitoring" its self-activity (via its evoked interoceptive state), as previously suggested (but not demonstrated) by classic experiments of volitional control of neural signals proposed by Eberhard E. Fetz (37), since these experiments necessarily require an exteroceptive sensory stimulus (auditory or visual) as a feedback cue to learn $(38)$.

The use of optoception also implies that it can be implemented as an independent sensory channel to control brain-computer interfaces. This idea has been recently demonstrated by Prsa et al. 2017 who showed that mice could use artificial optogenetic stimulation of S1 cortex as sensory feedback to accelerate the control of their own M1 neuronal activity (34), thereby presenting an opportunity for using optoception as a parallel information channel to perform brain-computer interfaces (38). Our results extend these observations to show that the cortex or subcortical regions and stimulating or silencing neurons could potentially be used as an additional sensory channel to introduce information to the brain.

Finally, given that a primary goal of optogenetics is to unveil the physiological function of a given cell type, our results emphasize the importance of researchers considering this factor in interpreting optogenetic experiments, given that animals could also learn from the brain stimulation per se.

\section{References and Notes}

1. A. D. Craig, How do you feel? Interoception: the sense of the physiological condition of the body. Nat Rev Neurosci. 3, 655-666 (2002).

2. S. S. Khalsa, R. C. Lapidus, Can Interoception Improve the Pragmatic Search for Biomarkers in Psychiatry? Front. Psychiatry. 7 (2016), doi:10.3389/fpsyt.2016.00121.

3. R. Hölzl, L.-P. Erasmus, A. Möltner, Detection, discrimination and sensation of visceral stimuli. Biological Psychology. 42, 199-214 (1996). 
4. R. Verrier, A. Calvert, B. Lown, Effect of posterior hypothalamic stimulation on ventricular fibrillation threshold. American Journal of Physiology-Legacy Content. 228, 923-927 (1975).

5. R. Romo, A. Hernández, A. Zainos, E. Salinas, Somatosensory discrimination based on cortical microstimulation. Nature. 392, 387-390 (1998).

6. K. A. Mazurek, M. H. Schieber, Injecting Instructions into Premotor Cortex. Neuron. 96, 1282-1289.e4 (2017).

7. M. Desmurget, K. T. Reilly, N. Richard, A. Szathmari, C. Mottolese, A. Sirigu, Movement Intention After Parietal Cortex Stimulation in Humans. Science. 324, 811-813 (2009).

8. Z. Wu, N. Zheng, S. Zhang, X. Zheng, L. Gao, L. Su, Maze learning by a hybrid braincomputer system. Sci Rep. 6, 31746 (2016).

9. G. D. Scala, J. Mana, W. J. Jacobs, A. G. Phillips, Evidence of Pavlovian Conditioned Fear Following Electrical Stimulation of the Periaqueductal Grey in the Rat I, 9.

10. S. Kumar, S. J. Black, R. Hultman, S. T. Szabo, K. D. DeMaio, J. Du, B. M. Katz, G. Feng, H. E. Covington, K. Dzirasa, Cortical Control of Affective Networks. Journal of Neuroscience. 33, 1116-1129 (2013).

11. C. Porrero, P. Rubio-Garrido, C. Avendaño, F. Clascá, Mapping of fluorescent proteinexpressing neurons and axon pathways in adult and developing Thy1-eYFP-H transgenic mice. Brain Research. 1345, 59-72 (2010).

12. S. S. Babl, B. P. Rummell, T. Sigurdsson, The Spatial Extent of Optogenetic Silencing in Transgenic Mice Expressing Channelrhodopsin in Inhibitory Interneurons. Cell Reports. 29, 1381-1395.e4 (2019).

13. O. Yizhar, O. Klavir, Reciprocal amygdala-prefrontal interactions in learning. Current Opinion in Neurobiology. 52, 149-155 (2018).

14. J. Courtin, F. Chaudun, R. R. Rozeske, N. Karalis, C. Gonzalez-Campo, H. Wurtz, A. Abdi, J. Baufreton, T. C. M. Bienvenu, C. Herry, Prefrontal parvalbumin interneurons shape neuronal activity to drive fear expression. Nature. 505, 92-96 (2014).

15. S. Zhao, J. T. Ting, H. E. Atallah, L. Qiu, J. Tan, B. Gloss, G. J. Augustine, K. Deisseroth, M. Luo, A. M. Graybiel, G. Feng, Cell type-specific channelrhodopsin-2 transgenic mice for optogenetic dissection of neural circuitry function. Nature Methods. 8, 745-752 (2011).

16. L. Prado, J. Luis-Islas, O. I. Sandoval, L. Puron, M. M. Gil, A. Luna, M. A. Arias-García, E. Galarraga, S. A. Simon, R. Gutierrez, Activation of Glutamatergic Fibers in the Anterior NAc Shell Modulates Reward Activity in the aNAcSh, the Lateral Hypothalamus, and Medial Prefrontal Cortex and Transiently Stops Feeding. J. Neurosci. 36, 12511-12529 
17. D. Pinault, The thalamic reticular nucleus: structure, function and concept. Brain Research Reviews. 46, 1-31 (2004).

18. E. Gil-Lievana, I. Balderas, P. Moreno-Castilla, J. Luis-Islas, R. A. McDevitt, F. Tecuapetla, R. Gutierrez, A. Bonci, F. Bermúdez-Rattoni, Glutamatergic basolateral amygdala to anterior insular cortex circuitry maintains rewarding contextual memory. Commun Biol. 3, 139 (2020).

19. H. E. Heffner, R. S. Heffner, Hearing ranges of laboratory animals. J. Am. Assoc. Lab. Anim. Sci. 46, 20-22 (2007).

20. B. Danskin, D. Denman, M. Valley, D. Ollerenshaw, D. Williams, P. Groblewski, C. Reid, S. Olsen, J. Waters, Optogenetics in Mice Performing a Visual Discrimination Task: Measurement and Suppression of Retinal Activation and the Resulting Behavioral Artifact. PLoS ONE. 10, e0144760 (2015).

21. M. M. Halassa, Z. Chen, R. D. Wimmer, P. M. Brunetti, S. Zhao, B. Zikopoulos, F. Wang, E. N. Brown, M. A. Wilson, State-Dependent Architecture of Thalamic Reticular Subnetworks. Cell. 158, 808-821 (2014).

22. C. Banuelos, B. S. Beas, J. A. McQuail, R. J. Gilbert, C. J. Frazier, B. Setlow, J. L. Bizon, Prefrontal Cortical GABAergic Dysfunction Contributes to Age-Related Working Memory Impairment. Journal of Neuroscience. 34, 3457-3466 (2014).

23. R. N. Walsh, R. A. Cummins, The open-field test: A critical review. Psychological Bulletin. 83, 482-504 (1976).

24. E. Choleris, A detailed ethological analysis of the mouse open field test: effects of diazepam, chlordiazepoxide and an extremely low frequency pulsed magnetic field. Neuroscience \& Biobehavioral Reviews. 25, 235-260 (2001).

25. D. Scheggia, F. Managò, F. Maltese, S. Bruni, M. Nigro, D. Dautan, P. Latuske, G. Contarini, M. Gomez-Gonzalo, L. M. Requie, V. Ferretti, G. Castellani, D. Mauro, A. Bonavia, G. Carmignoto, O. Yizhar, F. Papaleo, Somatostatin interneurons in the prefrontal cortex control affective state discrimination in mice. Nat Neurosci. 23, 47-60 (2020).

26. B. Y. Chow, X. Han, A. S. Dobry, X. Qian, A. S. Chuong, M. Li, M. A. Henninger, G. M. Belfort, Y. Lin, P. E. Monahan, E. S. Boyden, High-performance genetically targetable optical neural silencing by light-driven proton pumps. Nature. 463, 98-102 (2010).

27. E. H. Nieh, C. M. Vander Weele, G. A. Matthews, K. N. Presbrey, R. Wichmann, C. A. Leppla, E. M. Izadmehr, K. M. Tye, Inhibitory Input from the Lateral Hypothalamus to the Ventral Tegmental Area Disinhibits Dopamine Neurons and Promotes Behavioral Activation. Neuron. 90, 1286-1298 (2016).

28. A. Garcia, A. Coss, J. Luis-Islas, L. Puron-Sierra, M. Luna, M. Villavicencio, R. Gutierrez, Lateral Hypothalamic GABAergic Neurons Encode and Potentiate Sucrose's Palatability. Front. Neurosci. 14 (2021), doi:10.3389/fnins.2020.608047. 
29. R. W. Doty, Conditioned reflexes elicited by electrical stimulation of the brain in macaques. Journal of Neurophysiology (1965), doi:10.1152/jn.1965.28.4.623.

30. K. A. Mazurek, M. H. Schieber, How is electrical stimulation of the brain experienced, and how can we tell? Selected considerations on sensorimotor function and speech. Cognitive Neuropsychology. 36, 103-116 (2019).

31. G. A. Tabot, J. F. Dammann, J. A. Berg, F. V. Tenore, J. L. Boback, R. J. Vogelstein, S. J. Bensmaia, Restoring the sense of touch with a prosthetic hand through a brain interface. PNAS. 110, 18279-18284 (2013).

32. W. Penfield, T. Rasmussen, The Cerebral Cortex of Man: A Clinical Study of Localization of Function (Macmillian, New York, 1950).

33. Z. Sun, A. Schneider, M. Alyahyay, G. Karvat, I. Diester, Effects of Optogenetic Stimulation of Primary Somatosensory Cortex and Its Projections to Striatum on Vibrotactile Perception in Freely Moving Rats. eNeuro. 8 (2021), doi:10.1523/ENEURO.0453-20.2021.

34. G. Razran, The observable and the inferable conscious in current Soviet psychophysiology: Interoceptive conditioning, semantic conditioning, and the orienting reflex. Psychological Review. 68, 81-147 (1961).

35. R. A. Bevins, J. Besheer, Interoception and Learning: Import to Understanding and Treating Diseases and Psychopathologies. ACS Chem. Neurosci. 5, 624-631 (2014).

36. E. Ceunen, J. W. S. Vlaeyen, I. V. Diest, On the Origin of Interoception. Frontiers in Psychology. 7 (2016), doi:10.3389/fpsyg.2016.00743.

37. E. E. Fetz, Operant Conditioning of Cortical Unit Activity. Science. 163, 955-958 (1969).

38. A. C. Koralek, X. Jin, J. D. Long Ii, R. M. Costa, J. M. Carmena, Corticostriatal plasticity is necessary for learning intentional neuroprosthetic skills. Nature. 483, 331-335 (2012).

39. R. D. Wimmer, L. I. Schmitt, T. J. Davidson, M. Nakajima, K. Deisseroth, M. M. Halassa, Thalamic control of sensory selection in divided attention. Nature. 526, 705-709 (2015).

40. L. Vong, C. Ye, Z. Yang, B. Choi, S. Chua, B. B. Lowell, Leptin Action on GABAergic Neurons Prevents Obesity and Reduces Inhibitory Tone to POMC Neurons. Neuron. 71, 142-154 (2011).

41. E. Fonseca, V. de Lafuente, S. A. Simon, R. Gutierrez, Sucrose intensity coding and decision-making in rat gustatory cortices. eLife. 7, e41152 (2018).

Acknowledgments: We thank Professor Sidney A. Simon, Federico Bermudez-Rattoni, Pavel Rueda, and Román Rossi Pool for their thoughtful comments on this paper. To Mario GilMoreno for building homemade optrodes and to Alam Coss, Raul Sanchez Granados, and 
Aketzali Garcia for technical help with experimental work. We also thank Ricardo Gaxiola, Victor Manuel Garcia Gomez, and Fabiola Hernandez Olvera for invaluable animal care.

Funding: This project was supported in part by Productos Medix 3247, Cátedra Marcos Moshinsky, fundación Miguel Aleman Valdes, CONACyT CF63 PN464 (R.G.). M.L. performed rtCPP and frequency discrimination task. J. LI. and M.L. performed histological sections. J. LI analyzed data. B.F. and M.L. reviewed the paper. J. LI and R.G. wrote the paper

Competing interests: Authors declare no competing interests

Data and materials availability: materials and methods are available in the supplementary materials.

\section{Supplementary Materials:}

Materials and Methods

Figures S1-S6

Movies S1-S7 

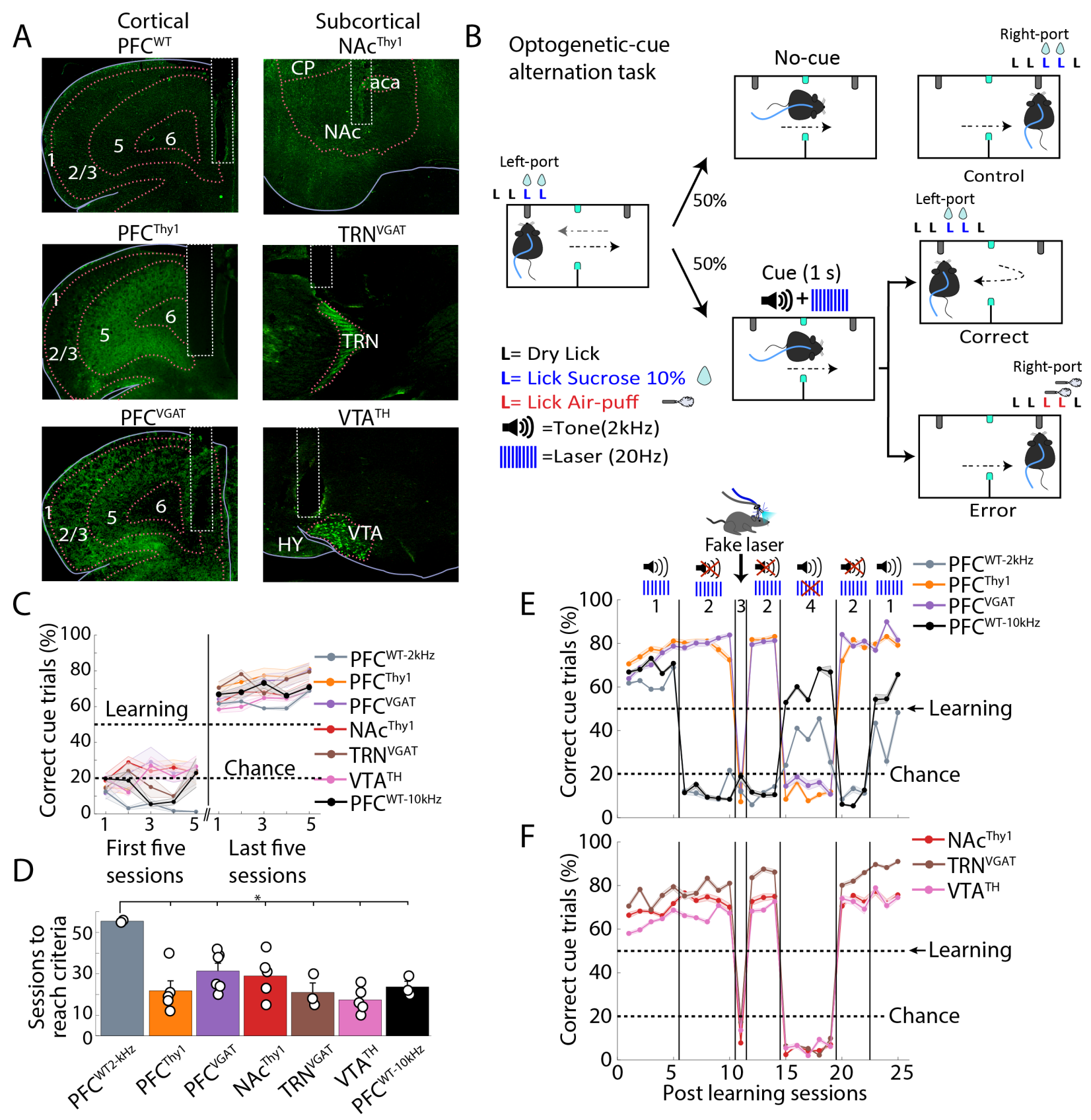

Fig 1. Mice learn to use optogenetic manipulations in various brain regions and cell types as a cue to guide behavior. (A) Representative images for fiber optics implantation and stimulation sites. Left pictures show unilateral optical fibers implanted in prefrontal cortices (PFCs) in wildtype $\left(\mathrm{PFC}^{\mathrm{WT}}\right)$, transgenic Thy1-ChR2 ( $\left.\mathrm{PFC}^{\mathrm{Thy}}\right)$, and VGAT-ChR2 (PFC $\left.{ }^{\mathrm{VGAT}}\right)$ mice. Right pictures show optical fibers in subcortical regions, including the nucleus accumbens (NAc) in Thy1-ChR2 (NAc ${ }^{\text {Thyl }}$ ), in the thalamic reticular nucleus (TRN) in VGAT-ChR2 (TRN ${ }^{\text {VGAT }}$ ), and the ventral tegmental area (VTA) in TH-Cre (VTA ${ }^{\mathrm{TH}}$ ) mice. (B) Schematic of the optogenetic-cue alternation task. In this task, mice had to alternate between two sippers to receive two drops of $10 \%$ sucrose from each one (additional licks were always dry). When the mice break the 
photobeam, located halfway between the two sippers (cyan squares), a combined cue consisting of a tone $(2 \mathrm{kHz})$ plus a laser $(20 \mathrm{~Hz}, 1 \mathrm{~s})$ was randomly delivered in $50 \%$ of trials. The combined cue instructed them to return to the previously rewarded port to be again rewarded. However, if they ignored the cue and licked at the opposite sipper, they received two air-puffs as a punishment. (C) Task performance in the initial first five and last five training sessions. Learning criteria (horizontal dashed line at 50\%) were reached when mice avoid punishment in at least $50 \%$ of cue trials in 5 consecutive sessions. Note that $\mathrm{PFC}^{\mathrm{WT}-10 \mathrm{kHz}}$ mice were trained with a more easily perceived auditory tone $10 \mathrm{kHz}$, than $2 \mathrm{kHz}$ tone that was barely perceptible to mice. (D) Sessions to reach the learning criteria. Each dot represents a mouse. (E) Task performance post-learning, in mice with optogenetic stimulation in PFC (block 1). The tone was removed in the subsequent 5 sessions (block 2). In block 3, mice were tested with a fake laser (a fiber optic shining light outside the skull). After reacquisition sessions with only the laser (second block 2), block 4 began, where the laser was replaced by the tone, which was the only cue. Finally, we repeated block 1, where the combined laser+tone cue was again delivered. (F) Similar to panel "E," but optogenetic stimulation was performed in NAc glutamatergic afferents (NAc ${ }^{\text {Thy } 1}$ ), in TRN GABAergic neurons $\left(\mathrm{TRN}^{\mathrm{VGAT}}\right.$ ), and midbrain dopaminergic neurons $\left(\mathrm{VTA}^{\mathrm{TH}}\right) .{ }^{*} p<0.01$ ANOVA Bonferroni post hoc, relative to $\mathrm{PFC}^{\mathrm{WT}}$
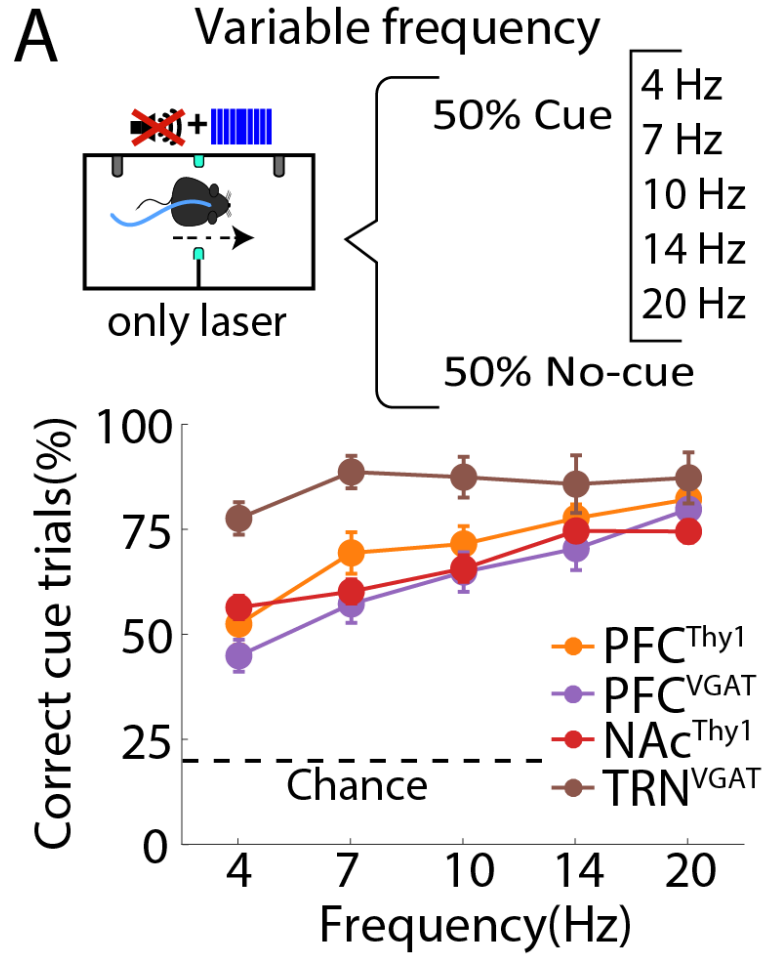
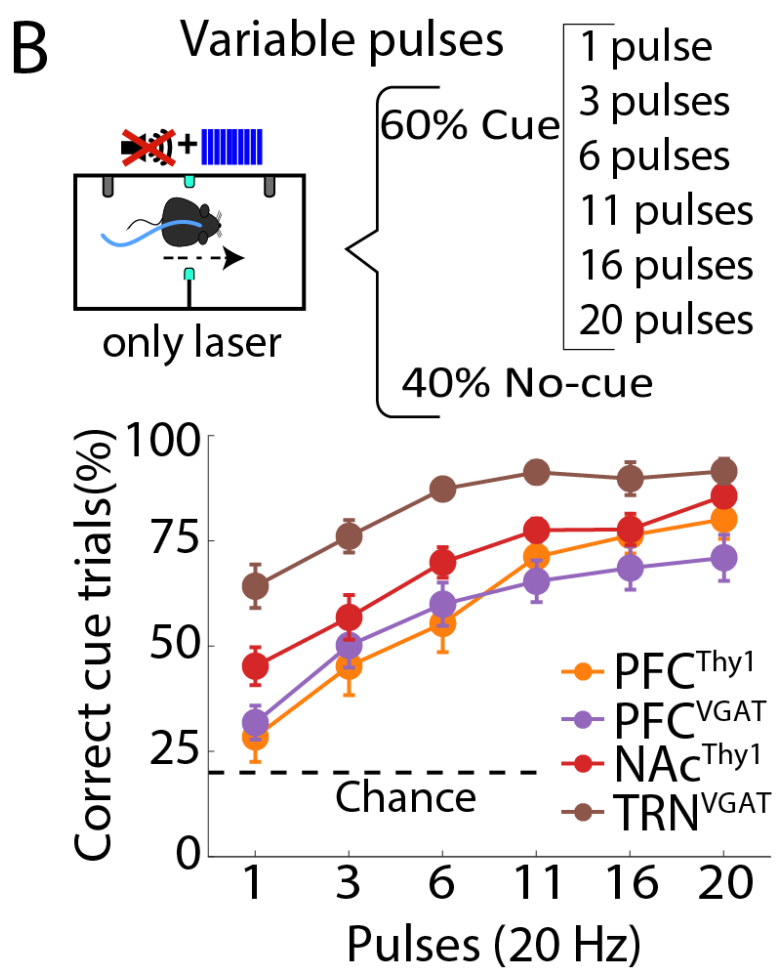

Fig. 2. Mice can use only optogenetic stimulation as a cue and generalize to other laser parameters. (A) Upper panel, schematics of the modified optogenetic-cue alternation task protocol where one out of five frequencies were randomly delivered in $50 \%$ of the trials. The bottom panel indicates correct cue trials (correct frequency trials / total frequency trials). WT mice were not tested in these task variants because they did not perceive the laser alone. (B) The upper panel depicts the structure of the modified pulse task variant. In this variant, one out of six laser pulses (from 1 to 20 ) was randomly delivered in $60 \%$ of trials. Below is shown the correct 
cue trials (correct pulse trials / total pulse trials). Note that TRN ${ }^{\mathrm{VGAT}}$ mice were so proficient on both tasks, perhaps because of their role in modulating attention (39).

A Frequency discrimination task

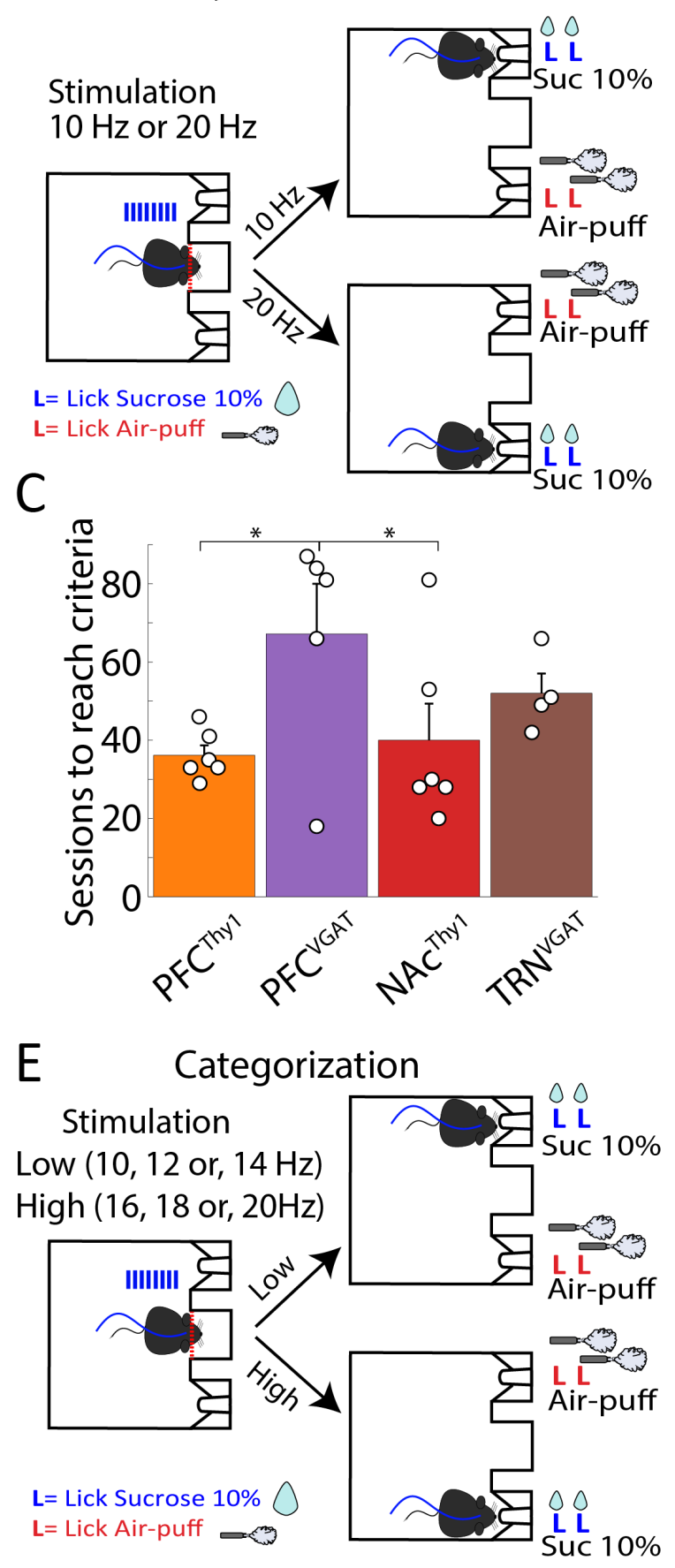

B

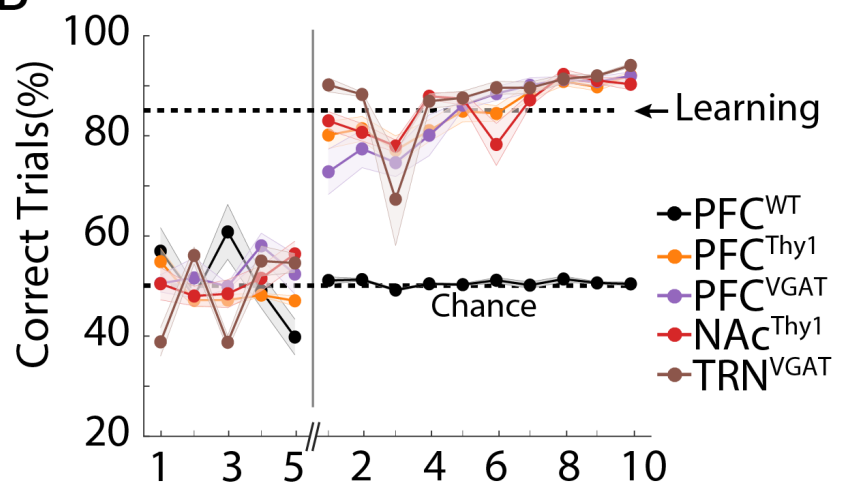

First five Last ten

D sessions

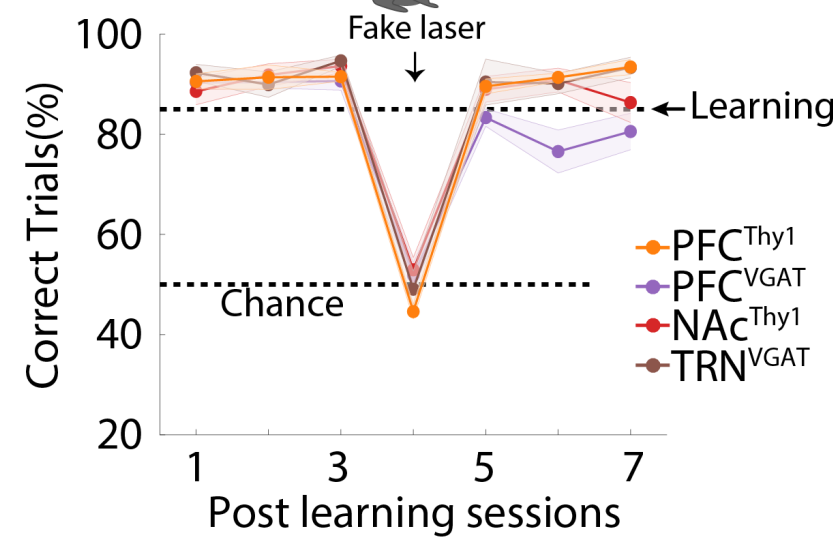

$\mathrm{F}$

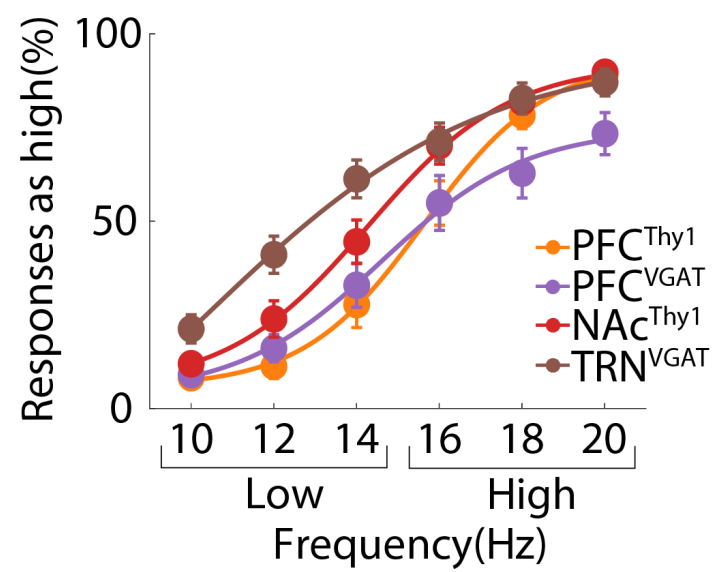

Fig. 3. Mice use different laser frequencies to distinguish two actions. (A) scheme of the frequency discrimination task. In this task, upon head entry in the central port (red dashed line), the laser was turned "on" $1 \mathrm{~s}$ at 10 or $20 \mathrm{~Hz}$ whereupon mice were required to lick in the lateral 
ports to receive either two drops of sucrose as a reward or two air-puffs as punishment (lateral ports were counterbalanced). (B) Correct trials were plotted for the initial five sessions, and the last ten sessions after subjects reached the learning criteria ( $85 \%$ correct trials in 3 consecutive sessions). The WT mice could not learn even after 90 training sessions. (C) The time needed to reach the learning criteria. (D) Task performance in subjects that learned the task before and after testing with a "fake laser" in which mice could see the blue light outside the skull but did not receive any optogenetic stimulation. (E) Structure of the generalization task, mice had to categorize 10,12, and $14 \mathrm{~Hz}$ frequencies as "low" and 16, 18, and $20 \mathrm{~Hz}$ as "high" by licking in the lateral ports. (F) Psychometric function showing that all group of mice chooses more the "high" port as the laser frequency increased. Note that as the laser frequency increases, mice choose more the "high" port, which confirms that they categorized the different laser frequencies. This procedure was counterbalanced across mice. ${ }^{*} p<0.05$, ANOVA.
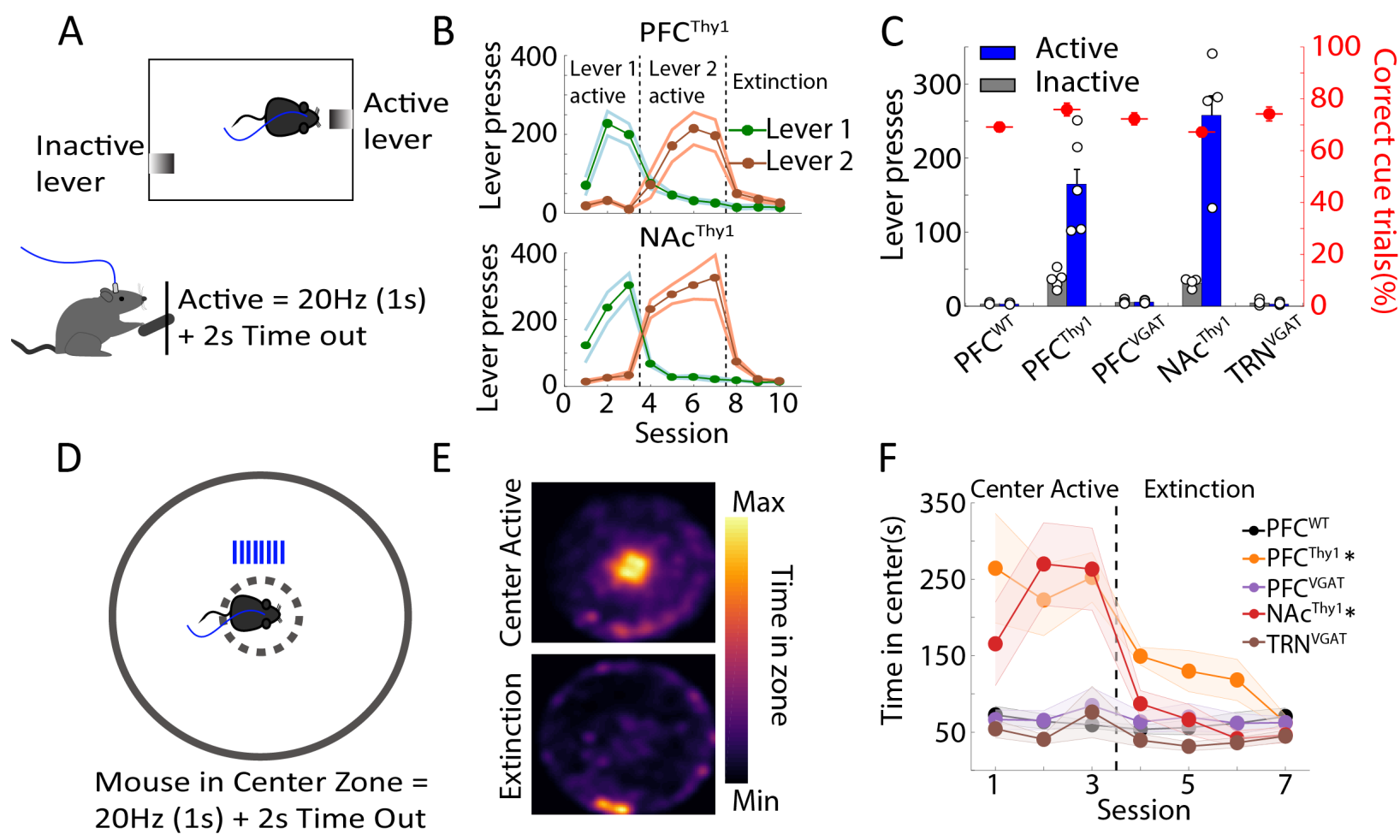

$\mathrm{E}$
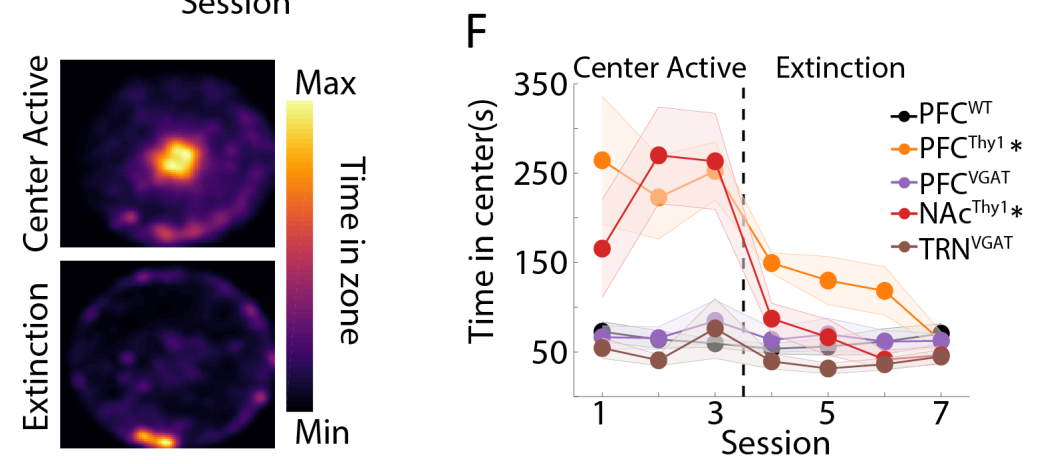

Fig. 4. Optoception can guide behavior irrespective if brain manipulations elicited rewarding effects or not. (A) Scheme of a lever self-stimulation task. Animals can trigger the delivery of laser stimulation by pressing the active lever $(20 \mathrm{~Hz}, 1 \mathrm{~s}+2 \mathrm{~s}$ of time out). The inactive lever was recorded but had no programmed consequence. (B) The number of lever presses across sessions. This shows that stimulation of $\mathrm{PFC}^{\text {Thy } 1}$ and NAc ${ }^{\text {Thy } 1}$ was rewarding, as indicated by the number of lever presses. After three sessions, the active lever was switched to inactive and tested for four additional sessions. Levers were counterbalanced across subjects. In the Extinction phase, both levers were inactive, and thus they did no laser stimulation was evoked. (C) Mean lever presses (excluding Extinction sessions). Small white dots indicate the number of mice tested. Overlapped is also shown their average performance (right axis) achieved in the optogenetic-cue alternation 
task (see solid red circles, Fig. 1C). (D) Open field center self-stimulation task. In this task, mice had to cross the center zone to receive laser stimulation $(20 \mathrm{~Hz}, 1 \mathrm{~s}+2 \mathrm{~s}$ of time out), note that no other reward or stimuli were delivered. (E) Representative heat map of a PFC ${ }^{\text {Thy } 1}$ mouse that crosses the center (Active) to self-stimulate. The bottom panel shows an extinction session of the same mouse. (F) The time spent in the center zone across sessions for all groups. ${ }^{*} \mathrm{p}<0.05$, ANOVA, Bonferroni post hoc, significantly different from $\mathrm{PFC}^{\mathrm{WT}}$ during active sessions.

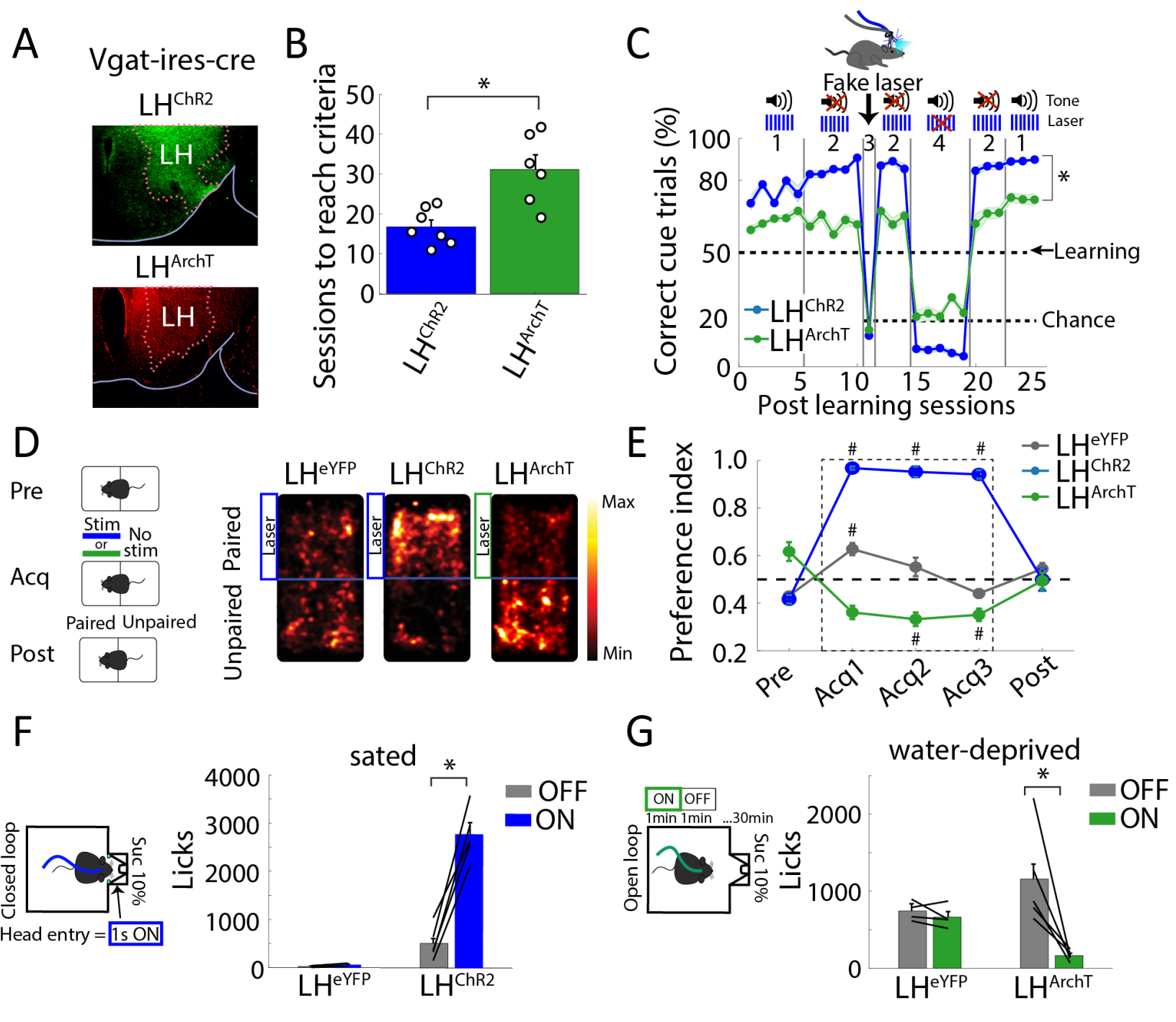

Fig. 5. Mice could use both activation or silencing of the same cell type as a sensory cue, even though they evoked opposing behavioral effects upon reward and feeding. (A) Histology of mice transfected with ChR2 or ArchT in Vgat-ires-cre mice (GABAergic neurons) of the Lateral Hypothalamus ( $\mathrm{LH}^{\mathrm{ChR} 2}$ or $\mathrm{LH}^{\mathrm{ArchT}}$, respectively). ChR2 was used for activation and ArchT for silencing LH GABAergic neurons. (B) The number of sessions required to reach the learning criterion. Each dot represents an individual mouse. ${ }^{*} p<0.001$ unpaired t-tests $(\mathbf{C})$ Correct trials in the presence of tone $(2 \mathrm{kHz})$ and/or laser in mice with fiber optics implanted in the LH region. Same conventions as in Figure 1E. ${ }^{*} p<0.001$ ANOVA two-way (transgenic mice $\mathrm{x}$ block) (D) Schematics of real-time conditioned place preference task (rtCPP). rtCPP consists of three phases. 
In the Pre-stimulation phase (Pre), the side that mice preferred is measured in the absence of stimulation. In the Acquisition phase (Acq), optogenetic stimulation is turned "on" when the mice entered and while it stayed on the paired side. Finally, in the Post-stimulation phase (Post), mice were not stimulated, and their preference index was measured. Right panels depict heat maps of a representative mouse on the acquisition phase. The control was a Vgat-ires-cre mouse only expressing the enhanced Yellow Fluorescent Protein $\left(\mathrm{LH}^{\mathrm{eYFP}}\right)$ (E) The preference index is the fraction of time spent on the paired side. A value above 0.5 means that the optogenetic stimulation is preferred, while a value below 0.5 means that stimulation was avoided. The dashed box indicates the acquisition sessions. \# $p<0.05$, ANOVA Bonferroni post hoc, relative to pre-test session. (F) Left, schematic of the closed-loop stimulation. In this protocol, sated $\mathrm{LH}^{\mathrm{ChR} 2}$ or $\mathrm{LH}^{\mathrm{eYFP}}$ mice were placed in a behavioral box with a sucrose sipper in the central port. Upon entry into the central port, the laser was triggered ( $1 \mathrm{~s}$ "on," $20 \mathrm{~Hz}+2 \mathrm{~s}$ time out, $473 \mathrm{~nm})$ in the LH $\mathrm{ChR}^{\mathrm{Ch}}$ mice. Right, the total sucrose intake in a closed-loop activation of LH GABAergic neurons. (G) Left, a protocol for open-loop silencing of LH GABAergic neurons in water-deprived $\mathrm{LH}^{\mathrm{ArchT}}$ or control $\mathrm{LH}^{\mathrm{eYFP}}$ mice. The green laser was turned "on" in blocks of 1 min with the laser (continuous pulse, at $532 \mathrm{~nm}$ ) and 1 min with no-laser "off." At right, the number of licks given while silencing LH GABAergic neurons in water-deprived mice. ${ }^{*} p<0.001$ paired t-test. 